\title{
Partial Resistance to Melampsora larici-populina Leaf Rust in Hybrid Poplars: Genetic Variability in Inoculated Excised Leaf Disk Bioassay and Relationship with Complete Resistance
}

\author{
A. Dowkiw, C. Husson, P. Frey, J. Pinon, and C. Bastien
}

First and fifth authors: Institut National de la Recherche Agronomique (INRA), Unité Amélioration, Génétique et Physiologie Forestières, av. de la Pomme de Pin, BP 20619 Ardon, F-45166 Olivet Cedex, France; and second, third, and fourth authors: Institut National de la Recherche Agronomique (INRA), Unité de Pathologie Forestière, F-54280 Champenoux, France. Accepted for publication 21 October 2002.

\begin{abstract}
Dowkiw, A., Husson, C., Frey, P., Pinon, J., and Bastien, C. 2003. Partial resistance to Melampsora larici-populina leaf rust in hybrid poplars: Genetic variability in inoculated excised leaf disk bioassay and relationship with complete resistance. Phytopathology 93:421-427.

Inoculated excised leaf disk technique allows decomposition of poplar partial resistance to Melampsora larici-populina leaf rust into key epidemiological components such as latent period (LP), uredinia number (UN), uredinia size (US), and spore production (SP) for a given $M$. larici-populina strain under controlled environmental conditions. Three hundred thirty-six genotypes from an interspecific Populus deltoides $\times P$. trichocarpa $\mathrm{F}_{1}$ progeny segregating for complete resistance to $M$. laricipopulina strain 93ID6 were inoculated with M. larici-populina strain

uredinia appearance dynamics did not yield additional information. Indirect assessment of SP by US scoring was reliable and easy to access compared with direct spore counting. UN was the only trait for which a doubling of the inoculum pressure level had a significant effect, leading to greater differentiation between genotypes. Consistent with previous studies is the clear relationship between presence of complete resistance against $M$. larici-populina strain 93ID6 and higher partial resistance to M. larici-populina strain 93CV1 (32\% longer LP, 76\% smaller UN, and $34 \%$ smaller US). In the subpopulation compatible with 93ID6, bimodal distribution of genotypic means for US suggested implication of a major gene inherited from the $P$. trichocarpa parent. Residual variation was noted for the three epidemiological components, suggesting that additional genes might condition these quantitative traits.
\end{abstract} 93CV1. This strain was able to infect the whole family, except few probable recombinants. LP, final UN, and final US after one infectious cycle proved to be relevant complementary descriptors of partial resistance. Area under the disease progress curve and other parameters of
Additional keywords: black cottonwood, defeated resistance genes, eastern cottonwood, exapted resistance, qualitative resistance, quantitative resistance, slow rusting.
Selection for resistance to leaf rust caused by the fungus Melampsora larici-populina Kleb. is an important challenge for poplar breeders. Severe economic losses reached $50 \%$ of annual growth in two 6-year-old cultivated stands through decreased photosynthesis efficiency, early defoliation, and increased susceptibility to other pests and diseases (10). This pathogen, native to Europe, successfully spread to Australia and New Zealand about 30 years ago $(28,29)$. Although scarce, it was detected in the United States about 10 years ago (21) and more recently in Iceland and Chile (J. Pinon, unpublished data).

Durable resistance to a disease is resistance that remains effective during its prolonged and widespread use in an environment favorable to the disease (7). Resistance of annual crop cultivars are regarded as durable when effective for 10 years despite wide exposure to the pathogen (14) but the useful lifetime for forest improved material exceeds several decades. During the past 20 years, European breeders succeeded in developing poplar cultivars carrying complete $M$. larici-populina resistances but they failed in achieving durable resistance. Poplar clones remained resistant to natural inoculum in the field during selection and testing (over 10 to 15 years), but resistance was overcome less than 5 years after wide commercial use due to emergence of virulent strains of the pathogen (20). Four major factors speed up

Corresponding author: C. Bastien; E-mail address: Catherine.Bastien@orleans.inra.fr

Publication no. P-2003-0130-01R

(c) 2003 The American Phytopathological Society the spread of newly emerged virulent M. larici-populina strains: (i) the compulsory sexual stage of the pathogen on larch favors high racial variability, (ii) airborne M. larici-populina urediniospores can spread long distances, (iii) M. larici-populina multiplies on poplar leaves for months, and (iv) poplars are generally cultivated in monoclonal stands, which implies no genetic diversity for resistance and a strong selection pressure on the pathogen.

Durability of new selected resistances will not be predictable until we gain insight into molecular and coevolutionary mechanisms underlying poplar-M. larici-populina interactions. Therefore, INRA research program puts emphasis on both complete and partial resistance by assessing their genetic variability, genetic determinism, and level of strain-specificity.

Optimal measurement techniques are necessary to understand the genetic architecture and variability of partial resistance on a large number of genotypes against a wide range of strains of the pathogen. Partial resistance is a form of incomplete resistance in which spore production (SP) is reduced even though the host plants are susceptible to infection (18). It delays the establishment of the fungus and reduces its reproduction. Even slight differences in these two components of infection greatly reduce the rate of disease development in the field because M. larici-populina is a polycyclic pathogen. Because it provides a good control of environmental factors and pathogen variability, inoculated leaf disk technique has been used by many authors to evaluate key epidemiological components such as latent period (LP), uredinia number (UN), and SP of M. larici-populina $(9,26)$. This technique allowed the discovery of a relationship between complete resistance to a 
given set of individual $M$. larici-populina strains and the expression of partial resistance components against another $M$. laricipopulina strain. A single gene cluster controls both complete resistance to $M$. larici-populina strain 93ID6 and partial resistance to M. larici-populina strain $93 \mathrm{CV} 1$ in five $P$. deltoides $\times P$. trichocarpa $\mathrm{F}_{1}$ progenies (8).

Our objectives were (i) a methodological discussion regarding leaf disk bioassays with focus on optimal evaluation of disease dynamics and SP and on the impact of inoculum pressure on expression of genetic variability, (ii) a precise estimation of the available genetic variability for several partial resistance components in a $P$. deltoides $\times P$. trichocarpa $\mathrm{F}_{1}$ progeny of large size, and (iii) a quantification of the genetic relationship pointed out by Lefèvre et al. (8) between expression of partial resistance components and presence of complete resistance genes. Original contributions of this study lie in more precise evaluation of SP compared with the 1-to-3 scale used by Lefèvre et al. (8) and in the evaluation of a fourfold larger full-sib $\mathrm{F}_{1}$ progeny, i.e., 336 genotypes versus 56 studied by Lefèvre et al. (8). Such large pedigree segregating for complete resistance to $M$. larici-populina strain 93ID6 allows insight into genotypic distributions for partial resistance to $M$. larici-populina strain $93 \mathrm{CV} 1$ both in presence and in absence of complete resistance to M. larici-populina strain 93ID6. Such control of background noise and pleiotropic/linkage effect of complete resistance genes favors detection of other genes effect on partial resistance.

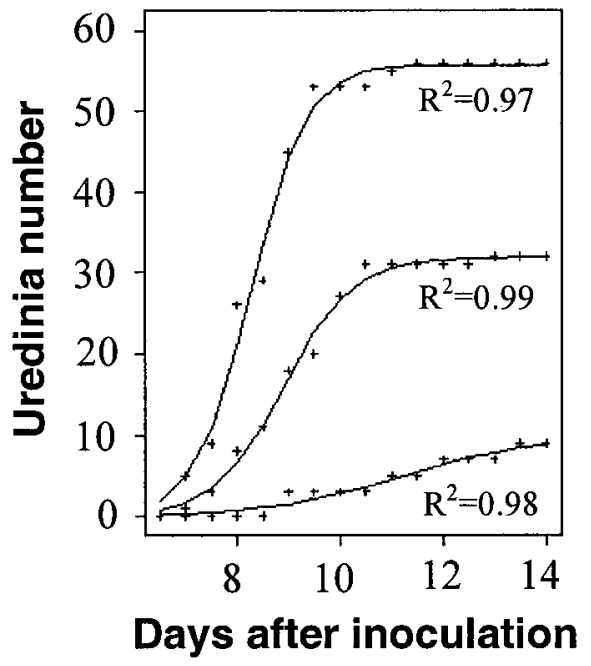

Fig. 1. Three representative examples of disease progress curves observed on Populus deltoides $\times$ P. trichocarpa full-sib clones excised leaf disks inoculated with Melampsora larici-populina single uredinial strain $93 \mathrm{CV} 1$ at 30 -mg/liter inoculum pressure and their associated coefficient of determination $\left(R^{2}\right)$ for adjustment to a logistic curve.

\section{MATERIALS AND METHODS}

Plant material. Poplar material consisted of 336 cloned $\mathrm{F}_{1}$ individuals from one interspecific $P$. deltoides $\times P$. trichocarpa cross. Methodological studies were conducted on random subsets. The two parents used for hybridization differ in their reaction to M. larici-populina. The $P$. deltoides female parent (73-028-62) showed complete or near-complete resistance to eight different strains of the pathogen studied so far, whereas the P. trichocarpa male parent (101-74) is compatible with all of them with varying levels of partial resistance (6). All $\mathrm{F}_{1}$ clones plus parents were grown from cuttings in 3 -liter pots containing $20 \%$ sand, $40 \%$ peat, and $40 \%$ ground bark mixture under glasshouse rust-free conditions. Natural daylight was supplemented for $16 \mathrm{~h} /$ day at $150 \mathrm{~W} \mathrm{~m}^{-2}$ minimum light intensity. The plants were watered and fertilized daily with a 15:10:15 fertilizing solution ( $1 \mathrm{~g} / \mathrm{liter})$.

Fungal material. The inoculum source consisted of urediniospores from single-uredinial $M$. larici-populina strain $93 \mathrm{CV} 1$. It was increased by spraying it on the abaxial side of $P$. xeuramericana highly susceptible cv. Robusta leaves floating upside down on distilled water in petri dishes under growth chamber controlled conditions $\left(19^{\circ} \mathrm{C}, 16-\mathrm{h}\right.$ photoperiod, $\left.30 \mu \mathrm{E} \mathrm{m}^{-2} \mathrm{~s}^{-1}\right)$. Urediniospores were collected 10 days later and stored in Eppendorf tubes at $4^{\circ} \mathrm{C}$.

Complete resistance assessment. All data concerning compatibility and incompatibility with $M$. larici-populina strain 93ID6 were taken from previous studies which concluded that there is 1:1 segregation for complete resistance to this strain (8).

Leaf disk bioassay general procedure. Inoculations were conducted on 3-cm-diameter excised leaf disks sampled from the fifth to the eight unrolled leaf below the apex because these leaves exhibit maximum sensitivity to the pathogen (27). The leaf disks were floated upside down on distilled water in polycarbonate sixwell cell culture plates. They were distributed in a complete block design, each $F_{1}$ genotype represented by one disk per block. Each parent was represented three times per block. A urediniospore suspension was sprayed over the disks with a hand atomizer. The inoculum suspension concentration was $30 \mathrm{mg} / \mathrm{liter}$ : $3 \mathrm{mg}$ of urediniospores in $100 \mathrm{ml}$ of distilled water with $10 \mathrm{mg}$ of agar. Sprayed suspension volume per leaf surface ratio was $20 \mathrm{ml} / \mathrm{m}^{2}$. The disks were kept for 14 days under growth chamber controlled conditions $\left(15^{\circ} \mathrm{C}, 16-\mathrm{h}\right.$ photoperiod, $30 \mu \mathrm{E} \mathrm{m}^{-2} \mathrm{~s}^{-1}$ ).

Description of disease progress. A random subset of $15 F_{1}$ individuals plus parents were studied in a five-complete-block design. UN was evaluated twice a day from day 5 to day 14 after inoculation. Two other parameters were derived from daily uredinia counting: LP, defined as the time after inoculation when the first sporulating uredinia appears, and area under the disease progress curve (AUDPC), calculated as

$$
\mathrm{AUDPC}=\sum_{1} \frac{\left(y_{i}+y_{i-1}\right) \times 0.5}{2}
$$

where $y_{i}$ is the number of uredinia the $i$ th half-day after inoculation (modified from literature citation 24).

TABLE 1. Observed general mean, range of variation (clonal means), estimated broad sense heritabilities $\left(H^{2}\right)$, and correlations for disease progress curve parameters $^{\mathrm{a}}$

\begin{tabular}{|c|c|c|c|c|c|c|c|}
\hline Trait & $\begin{array}{l}\text { Mean } \pm 95 \% \\
\text { confidence interval }\end{array}$ & $\begin{array}{l}\text { Range of } \\
\text { variation }\end{array}$ & $H^{2}(\%)$ & \multicolumn{4}{|c|}{$\begin{array}{l}\text { Pearson's correlation coefficient at the leaf disk level (under diagonal) an } \\
\text { at the clonal mean level (above diagonal) }\end{array}$} \\
\hline LP & $8.0 \pm 0.3$ & $6.9-9.6$ & 63 & & -0.81 & 0.86 & -0.89 \\
\hline$T_{i}$ & $9.5 \pm 0.3$ & $8.1-11.2$ & 59 & 0.63 & -0.61 & & -0.95 \\
\hline AUDPC & $159 \pm 22$ & $33-294$ & 69 & -0.72 & 0.96 & -0.74 & \\
\hline $\mathrm{DPR}_{\max }$ & $0.088 \pm 0.019$ & $0.045-0.156$ & n.s. & 0.23 & -0.31 & -0.10 & -0.23 \\
\hline
\end{tabular}

${ }^{a}$ Latent period (LP), asymptotic uredinia number at the end of experimentation $\left(\mathrm{UN}_{\text {asympt }}\right)$, date at the inflexion point $\left(T_{i}\right)$, area under disease progress curve (AUDPC), and maximum disease progress rate $\left(\mathrm{DPR}_{\max }\right)$ estimated on 15 Populus deltoides $\times$ P. trichocarpa excised leaf disks inoculated with Melampsora larici-populina single uredinial strain $93 \mathrm{CV} 1$ at $30-\mathrm{mg} /$ liter inoculum pressure in a five complete block design. $\mathrm{n} . \mathrm{s}$. $=$ not significant. 
Estimation of uredinia SP. Two experiments were conducted in five-complete-block designs on the whole $F_{1}$ family plus parents.

One experiment aimed at comparing uredinia SP (direct measurement) through urediniospore sucking and counting with uredinia size scoring on a 1-to-5 scale ( $\left.\mathrm{US}_{\text {scale }}\right)$. The $336 \mathrm{~F}_{1}$ genotypes plus parents were studied. All disks were evaluated 14 days after inoculation for $\mathrm{US}_{\text {scale }}$ before SP was measured on $207 \mathrm{~F}_{1}$ disks. These 207 disks were chosen on the basis of their $\mathrm{US}_{\text {scale }}$ in order to allow the realization of two to three urediniospore suspensions

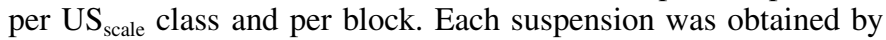
sucking up urediniospores from 30 uredinia from several disks of same $\mathrm{US}_{\text {scale. }}$ A vacuum flask and pump system was used to suck urediniospores into $100 \mathrm{ml}$ of saline solution (Coulter Isoton II; Beckman Coulter, Fullerton, CA). A particle counting machine (Coulter Z1; Beckman Coulter) was used to measure urediniospore concentration $(C$, urediniospores per liter). Average SP per uredinia was calculated for each suspension as $\mathrm{SP}=C \times 0.1 / N$.

Another experiment aimed at comparing $\mathrm{US}_{\text {scale }}$ with uredinia size measured by image analysis of leaf disk picture captured by video camera $\left(\mathrm{US}_{\text {image }}\right)$. All disks were evaluated 14 days after inoculation for $\mathrm{US}_{\text {scale }}$. Then, a subset of five $\mathrm{F}_{1}$ disks per $\mathrm{US}_{\text {scale }}$
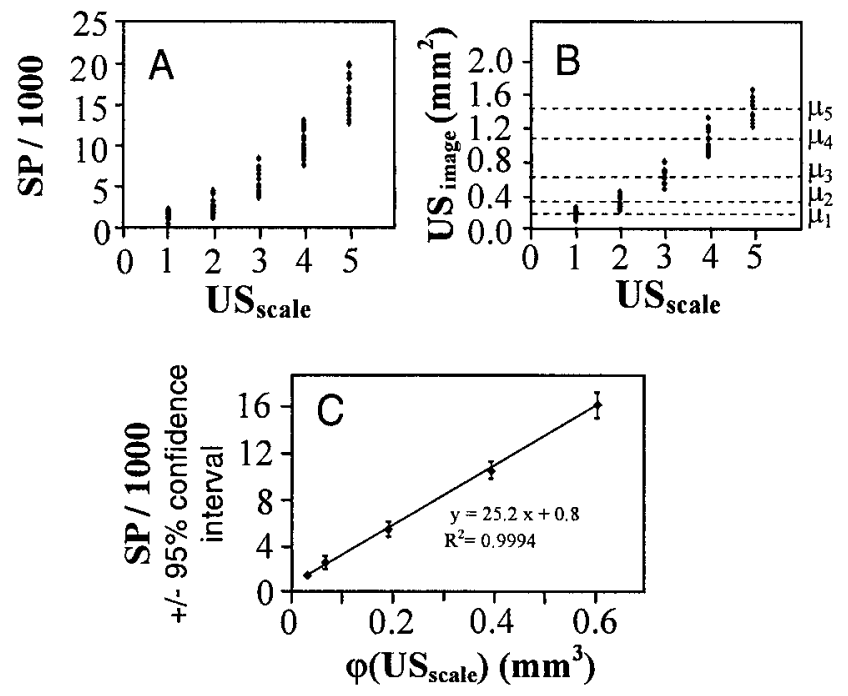

Fig. 2. Relationships observed 14 days after inoculation of Populus deltoides $\times$ P. trichocarpa full-sib clones with Melampsora larici-populina single uredinial strain 93CV1 at 30-mg/liter inoculum pressure level between A, uredinia average spore production (SP) and uredinia size score (US scale $_{\text {s. }}$, each point representing a pool of several excised leaf disks of same $\mathrm{US}_{\text {scale }} ; \mathbf{B}$, $\mathrm{US}_{\text {scale }}$ and uredinia size measured by image analysis $\left(\mathrm{US}_{\text {image }}\right)$, each point representing one excised leaf disk; and $\mathbf{C}$, uredinia average SP and uredinia volume estimation $\left(\varphi\left(\mathrm{US}_{\text {scale }}\right)\right)$. C, Derives from figure $\mathbf{A}$, where $\mathrm{SP}$ has been averaged for each $\mathrm{US}_{\text {scale }}$ class and where $\mathrm{US}_{\text {scale }}$ has been transformed into $\varphi\left(\mathrm{US}_{\text {scale }}\right), \varphi$ being defined as $\varphi(x)=2 /\left(3 \pi^{0.5}\right) \mu_{x}^{1.5}$ for $x \in(1,2,3,4,5)$, based on the hypothesis of a half-ball uredinia shape with $\mu_{x}$ the observed $U_{\text {image }}$ mean for $\mathrm{US}_{\text {scale }}=x$ in figure $\mathbf{B}$. class and per block, i.e., 75 disks in total were also evaluated for $\mathrm{US}_{\text {image. }}$

Estimation of inoculum pressure effect. Two experiments were conducted at the same time in the same growth chamber on the same random set of $69 \mathrm{~F}_{1}$ genotypes in 10-complete-block designs. A twofold 60-mg/liter inoculum concentration was used in the second experiment in order to foresee the effect of contrasted inoculum pressures on partial resistance components assessment. LP was measured on a half day basis, UN and $\mathrm{US}_{\text {scale }}$ were measured 13 and 14 days after inoculation, respectively. In order to better assess inoculum pressure, five 3 -cm-diameter petri dishes containing solid agar ( $20 \mathrm{~g} /$ liter) were scattered in each of the five blocks of each experiment prior to inoculation. Urediniospore number $(\mathrm{SN})$ was then measured in each petri dish with a microscope.

Estimation of genetic variability for partial resistance components. The $336 \mathrm{~F}_{1}$ genotypes plus parents were evaluated for LP on a half day basis, UN 13 days after inoculation, and $\mathrm{US}_{\text {scale }} 14$ days after inoculation in a 5-complete-block design. In order to better assess inoculum pressure, 153 -cm-diameter petri dishes containing solid agar ( $20 \mathrm{~g} / \mathrm{liter})$ were scattered in each of the five blocks of each experiment prior to inoculation. SN was then measured in each petri dish with a microscope.

Data analysis. Data were analyzed using S-plus software version 3.4 release 1 for Sun SPARC (Statistical Sciences, MathSoft Inc., Seattle, WA). A logistic function (equation 2) was used to summarize disease progress curves into a finite number of simple parameters.

$$
U N=\frac{U N_{\text {asympt }}}{1+\exp \left(\frac{T_{i}-t}{k}\right)}
$$

where $\mathrm{UN}_{\text {asympt }}$ is the asymptotic final $\mathrm{UN}, T_{i}$ is the date at the inflexion point, and $k$ is a scale parameter. The maximum relative

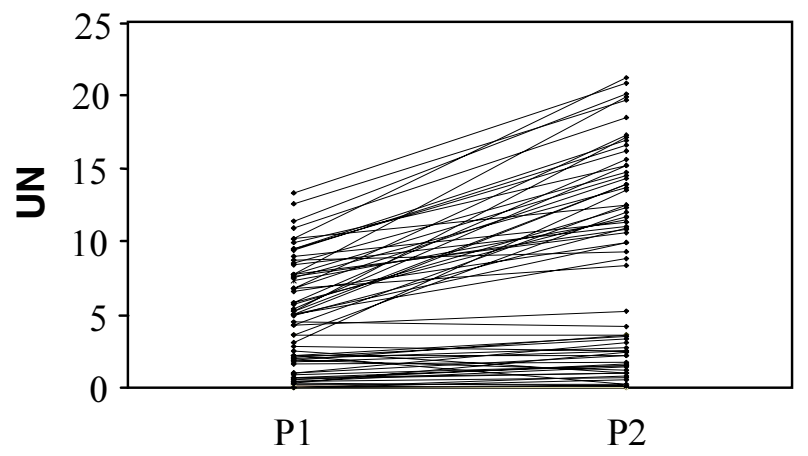

Fig. 3. Evolution of genotypic means for uredinia number (UN) 13 days after inoculation in a random subset of 69 Populus deltoides $\times$ P. trichocarpa full-sib clones inoculated with Melampsora larici-populina single uredinial strain $93 \mathrm{CV} 1$ with a doubling of the inoculum pressure level $(\mathrm{P} 1=30 \mathrm{mg} / \mathrm{liter}$, $\mathrm{P} 2=60 \mathrm{mg} /$ liter). Genotypic means are averaged across 10 blocks for each inoculum pressure level.

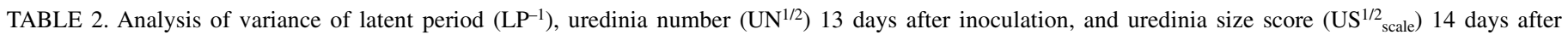

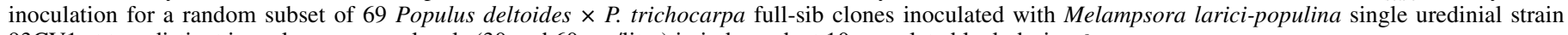
$93 \mathrm{CV} 1$ at two distinct inoculum pressure levels (30 and $60 \mathrm{mg} / \mathrm{liter})$ in independent 10 complete block designs ${ }^{\mathrm{a}}$

\begin{tabular}{|c|c|c|c|c|c|c|c|c|c|c|c|c|}
\hline \multirow[b]{2}{*}{ Source of variation } & \multicolumn{4}{|c|}{$\mathrm{LP}^{-1}$} & \multicolumn{4}{|c|}{$\mathrm{UN}^{1 / 2}$} & \multicolumn{4}{|c|}{$\mathrm{US}^{1 / 2}$ scale } \\
\hline & df & MS & $F$ & $P$ & df & MS & $F$ & $P$ & df & MS & $F$ & $P$ \\
\hline Inoculum pressure $(\mathrm{P})$ & 1 & 0.0012 & 4.0 & 0.059 & 1 & 136 & 19.0 & $*$ & 1 & 0.01 & 0.04 & 0.852 \\
\hline Genotype (G) & 67 & 0.0022 & 18.2 & $*$ & 68 & 30 & 44.5 & $*$ & 67 & 0.88 & 20.91 & $*$ \\
\hline Block B within P & 18 & 0.0003 & 2.1 & 0.004 & 18 & 6 & 8.7 & $*$ & 18 & 0.14 & 3.25 & $*$ \\
\hline $\mathrm{G} \times \mathrm{P}$ & 62 & 0.0002 & 1.3 & 0.055 & 68 & 2 & 2.8 & $*$ & 62 & 0.05 & 1.07 & 0.328 \\
\hline Error & 879 & 0.0001 & & & 1,218 & 1 & & & 893 & 0.04 & & \\
\hline
\end{tabular}

${ }^{\text {a }} P$ values below $1.10^{-3}$ appear as an asterisk. MS $=$ mean squares. 
disease progress rate $\left(\mathrm{DPR}_{\max }\right)$ was calculated as $\mathrm{DPR}_{\max }=(k \times$ $\left.\mathrm{UN}_{\text {asympt }}\right)^{-1}$. Disease progress curve parameters were estimated for each inoculated leaf disk by the least squares criterion adjustment and the Gauss-Marquardt algorithm.

Prior to any analysis of variance (ANOVA), data were corrected for normality using Box and Cox transformation (1). The ANOVA model used to test inoculum pressure effect was

$$
Y_{i j k}=\mu+P_{i}+B_{j(i)}+G_{k}+G P_{i k}+\varepsilon_{i j k}
$$

where $P$ is the inoculum pressure effect (fixed), $B$ is the block effect nested in $P$ (random), $G$ is the genotype effect (random), and $G P$ is the genotype by inoculum pressure interaction effect (random).

The ANOVA model used to assess genetic variability on the whole $\mathrm{F}_{1}$ and to test compatibility with 93ID6 effect was

$$
Y_{i j k}=\mu+B_{i}+C_{j}+G_{k(j)}+\varepsilon_{i j k}
$$

where $B$ is the block effect (random), $C$ is the compatibility with 93ID6 effect (fixed), and $G$ is the genotype effect (random).

The ANOVA model used to assess genetic variability expressed at a given inoculum pressure level in a given compatibility group was

$$
Y_{i j}=\mu+B_{i}+G_{j}+\varepsilon_{i j}
$$

Restricted maximum likelihood estimates of variances of all random factors $\left(\sigma_{B}^{2}, \sigma_{G}^{2}\right.$, and $\left.\sigma_{\varepsilon}^{2}\right)$ were then computed. Broad sense heritabilities were estimated as $H^{2}=\sigma_{G}^{2} / \sigma_{\text {Total }}^{2}$, with $\sigma_{\text {Total }}^{2}=$ $\sigma_{B}^{2}+\sigma_{G}^{2}+\sigma_{\varepsilon}^{2}$.

\section{RESULTS}

Disease progress. Appearance of the first sporulating uredinia occurred between the fifth and the tenth day after inoculation (clonal means) and all disease progress curves reached a plateau

TABLE 3. Observed range of variation (clonal means), observed general mean, and estimated broad sense heritability $\left(H^{2}\right)$ of uredinia number (UN) 13 days after inoculation for a random subset of 69 Populus deltoides $\times P$. trichocarpa full-sib clones inoculated with Melampsora larici-populina

\begin{tabular}{|c|c|c|c|c|}
\hline & & & & \\
\hline & & & & \\
\hline & P1 & P2 & P1 & P2 \\
\hline Range & $3.1-13.3$ & $3.6-21.2$ & $0.0-4.5$ & $0.0-4.2$ \\
\hline Mean & 7.3 & 13.6 & 1.2 & 1.6 \\
\hline$H^{2}(\%)$ & 20.0 & 21.9 & 32.0 & 32.9 \\
\hline
\end{tabular}
single uredinial strain 93CV1 at two distinct inoculum pressure levels $(\mathrm{P} 1=$ $30 \mathrm{mg} /$ liter; P2 = $60 \mathrm{mg} /$ liter) in independent 10-complete-block designs ${ }^{\mathrm{a}}$

${ }^{a}$ Data are presented separately for the 39 clones compatible (C) with $M$. larici-populina strain 93ID6 and for the 30 clones incompatible (I) with this strain. less than 14 days after inoculation. The logistic function fitted data from all studied disks with a determination coefficient $\left(R^{2}\right)$ greater than $95 \%$ (Fig. 1). $\mathrm{DPR}_{\max }$, which represents the relative speed at which the asymptotic UN is reached, showed no significant genetic variation. All other descriptors showed significant genetic variation and good heritabilities (Table 1). They were also highly correlated to each other. The less correlated traits were LP and $\mathrm{UN}_{\text {asympt }}$, i.e., the starting point in time and the final UN.

Uredinia SP. There was wide variation for SP ranging from 500 to 20,000 urediniospores per uredinia. Despite some intra $\mathrm{US}_{\text {scale }}$ class variability for SP, mostly due to block effects, there was a 0.96 Spearman's rank correlation coefficient between $\mathrm{US}_{\text {scale }}$ and SP. The relationship between the two traits was polynomial (Fig. 2A). Nonlinearity of this relationship may result from subjective nonlinear definition of $\mathrm{US}_{\text {scale }}$ classes which are based on surface area approximation. Quantification of the relationship between $\mathrm{US}_{\text {scale }}$ and $\mathrm{US}_{\text {image }}$ (Fig. 2B) allowed conversion of $\mathrm{US}_{\text {scale }}$ into average uredinia volume measured on the metric scale. Under the hypothesis of a half-ball uredinia shape, the conversion function $\varphi$ was defined as $\varphi(x)=2 /\left(3 \pi^{0.5}\right) \mu_{x}{ }^{1.5}$ for $x \in(1,2,3,4,5)$ with $\mu_{x}$ the observed $\mathrm{US}_{\text {image }}$ mean for $\mathrm{US}_{\text {scale }}=x$. The relationship between $\varphi\left(\mathrm{US}_{\text {scale }}\right)$ and SP showed very good linearity (Fig. 2C), thus demonstrating the linear link between SP and uredinia volume.

Inoculum pressure and genetic variability for partial resistance. Averaged SN counting in petri dishes, i.e., $56(\mathrm{SD}=18)$ and $112(\mathrm{SD}=35)$, confirmed that inoculum pressure in the high pressure experiment was actually twice that of the low pressure experiment. Even though all $\mathrm{F}_{1}$ genotypes are assumed compatible with strain $93 \mathrm{CV} 1$, four genotypes behaved as completely resistant under 30-mg/liter inoculum pressure level with one of them remaining at $\mathrm{UN}=0$ under $60-\mathrm{mg} / \mathrm{liter}$ inoculum pressure level. Regarding the expression of partial resistance components, neither LP nor $\mathrm{US}_{\text {scale }}$ were significantly affected by a doubling of the inoculum pressure. UN was the only trait for which there was significant inoculum pressure and inoculum pressure by genotype interaction effects (Table 2). However, there was a 0.89 Spearman's rank correlation coefficient between UN genotypic means for the two experiments. Most of the significant interaction effect came from a change in means dispersal, the most susceptible genotypes produced more uredinia under doubled inoculum pressure, whereas the most resistant ones remained stable. Two groups of genotypes clearly separated out under high inoculum pressure (Fig. 3). One group showed low and similar UN for both inoculum pressures. This group is mostly composed of genotypes incompatible with $M$. larici-populina strain 93ID6 (Table 3). The other group, strictly composed of genotypes compatible with these two strains, showed significantly higher UN under twofold inoculum pressure (Table 3). Separate analysis of UN variability expression in the compatible versus incompatible with strain 93ID6 subpopulations did not reveal significant increase of intra-subpopulation heritabilities under high inoculum pressure (Table 3).

Genetic variability for partial resistance components. Averaged SN counting in petri dishes was $74(\mathrm{SD}=19)$. Forty-nine genotypes behaved as completely resistant to strain 93CV1. High genotypic variability was observed for all partial resistance com-

TABLE 4. Analysis of variance of latent period $\left(\mathrm{LP}^{-2}\right)$, uredinia number $\left(\mathrm{UN}^{1 / 2}\right) 13$ days after inoculation, and uredinia size score (US ${ }^{1 / 2}$ scale $) 14$ days after

\begin{tabular}{|c|c|c|c|c|c|c|c|c|c|c|c|c|}
\hline \multirow[b]{2}{*}{$\underline{\text { Source of variation }}$} & \multicolumn{4}{|c|}{$\mathrm{LP}^{-2}$} & \multicolumn{4}{|c|}{$\mathrm{UN}^{1 / 2}$} & \multicolumn{4}{|c|}{$\mathrm{US}^{1 / 2}$ scale } \\
\hline & df & MS & $F$ & $P$ & df & MS & $F$ & $P$ & df & MS & $F$ & $P$ \\
\hline Block B & 4 & 0.000130 & 29.8 & * & 4 & 28.1 & 44.9 & * & 4 & 0.09 & 2.3 & 0.052 \\
\hline Compatibility group (C) & 1 & 0.008680 & 570.9 & * & 1 & $1,547.6$ & 398.9 & * & 1 & 17.51 & 43.0 & * \\
\hline Error & 929 & 0.000004 & & & 1,340 & 0.6 & & & 918 & 0.04 & & \\
\hline
\end{tabular}
inoculation for 336 Populus deltoides $\times$ P. trichocarpa full-sib clones inoculated with Melampsora larici-populina single uredinial strain 93CV1 at 30-mg/liter inoculum pressure level in a five-complete-block design ${ }^{\mathrm{a}}$

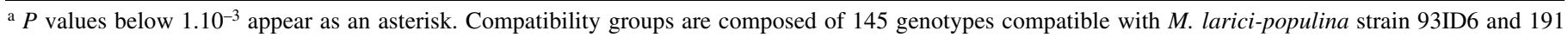
genotypes incompatible with this strain, respectively. MS = mean squares. 
ponents. Compatibility with strain 93ID6 had a significant effect on the expression of LP, UN, and $\mathrm{US}_{\text {scale }}$ (Table 4). Genotypes incompatible with this strain showed longer LP $(+32 \%$ on average), lower UN ( $-76 \%$ on average), and lower $\mathrm{US}_{\text {scale }}(-34 \%$ on average) (Fig. 4). Clonal variability pattern was also affected by compatibility with strain 93ID6.

In the compatible group, genotypes showed significant clonal variation mostly for UN and $\mathrm{US}_{\text {scale }}$ (Fig. 4; Table 5). For this last component, genotypes clearly split into two classes with a twofold difference in SP (Fig. 4). Correlations between LP and both UN and $\mathrm{US}_{\text {scale }}$ were significant but moderate (Fig. 5). No clear relationship was observed between UN and $\mathrm{US}_{\text {scale }}$ (Fig. 5).

In the incompatible group, LP variation range was 3 days longer (Fig. 4), contributing to higher heritability estimate (Table 5). The high correlations observed between LP and both UN and $\mathrm{US}_{\text {scale }}$ showed triangular patterns: genotypes with long LP had low UN and low $\mathrm{US}_{\text {scale, }}$, whereas genotypes with short LP showed a great range of variability for the other two components (Fig. 5). A closer relationship between $\mathrm{UN}$ and $\mathrm{US}_{\text {scale }}$ was observed in this group (Fig. 5).

\section{DISCUSSION}

Several authors pointed out the need for precise standardized protocols for $M$. larici-populina partial resistance assessments because many factors such as temperature, light, leaf maturity, shoot age, and hyperparasites have significant effects on its expression $(5,25,27)$. Definition of such protocols represents a prerequisite for the study of genetic determinism of $M$. larici-populina partial resistance and its strain-specificity. Thanks to good control of environmental conditions and genetic variability of the pathogen, this study confirms that leaf disk bioassays make it possible to access and quantify monocyclic variables that are key parameters of polycyclic disease progress. A large $P$. deltoides $\times$ $P$. trichocarpa $\mathrm{F}_{1}$ of 336 genotypes showing 1:1 segregation for complete resistance to $M$. larici-populina strain 93ID6 and where no complete resistance to $M$. larici-populina strain 93CV1 had been observed was evaluated for partial resistance to this last strain in excised leaf disk bioassays.

Methodological discussion. Daily UN counting did not appear to yield more information to discriminate genotypes than LP and final UN counting 13 days after inoculation. The date at the inflexion point $\left(T_{i}\right)$ was highly correlated with LP but less heritable. $\mathrm{DPR}_{\text {max }}$, the maximum relative speed at which disease increases, did not show significant variability in the studied material. AUDPC was fully determined by both the starting point of disease progress in time (LP) and the final UN ( $\left.\mathrm{UN}_{\text {asympt }}\right)$.

Uredinia SP was evaluated 14 days after inoculation using either direct measurement technique, i.e., urediniospores collection and counting (SP), or two indirect assessment techniques, i.e., uredinia size scoring using a five class ranking scale $\left(\mathrm{US}_{\text {scale }}\right)$ or uredinia size assessment using image analysis $\left(\mathrm{US}_{\text {image }}\right)$. Of the three techniques, urediniospores collection and counting is obviously the most repeatable but it is definitely too time consuming for large-scale experiments. Should the simple linear relationship we established between uredinia volume and uredinia SP be invariable, it would then be possible to access precise SP estimates through uredinia surface area measurements. This relationship is probably the same for any $M$. larici-populina strain in the studied $\mathrm{F}_{1}$ but it should be re-established for use in other progenies. By contrast, there is much probability for the relationship between $\mathrm{US}_{\text {scale }}$ and $\mathrm{US}_{\text {image }}$ to be highly variable from one mono-strain experiment to the other, even in the studied $F_{1}$, because definition of the five $\mathrm{US}_{\text {scale }}$ classes depends on the observed range of variation for this trait. Re-establishment of this relationship should be recommended on a sample of genotypes in each new experiment. Precise absolute estimates of SP would be highly valuable for

TABLE 5. Estimated broad sense heritabilities $\left(H^{2}\right)$ of latent period (LP), uredinia number (UN) 13 days after inoculation, and uredinia size score $\left(\mathrm{US}_{\text {scale }}\right) 14$ days after inoculation for 336 Populus deltoides $\times$ P. trichocarpa full-sib clones inoculated with Melampsora larici-populina single uredinial strain 93CV1 at 30-mg/liter inoculum pressure level in a five-complete-block design $^{\mathrm{a}}$

\begin{tabular}{ccccccccc}
\hline & \multicolumn{2}{c}{$\mathrm{LP}$} & & \multicolumn{2}{c}{$\mathrm{UN}$} & & \multicolumn{3}{c}{$\mathrm{US}_{\text {scale }}$} \\
\cline { 2 - 3 } \cline { 7 - 8 } & $\mathrm{C}$ & $\mathrm{I}$ & & $\mathrm{C}$ & $\mathrm{I}$ & & $\mathrm{C}$ & $\mathrm{I}$ \\
\hline$H^{2}(\%)$ & 26.0 & 46.2 & & 25.1 & - & & 76.8 & 57.0 \\
\hline
\end{tabular}

${ }^{a}$ Data are presented separately for the 145 clones compatible (C) with $M$. larici-populina strain 93ID6 and for the 191 clones incompatible (I) with this strain. No Box-Cox transformation for normality was found for UN in the incompatible group.

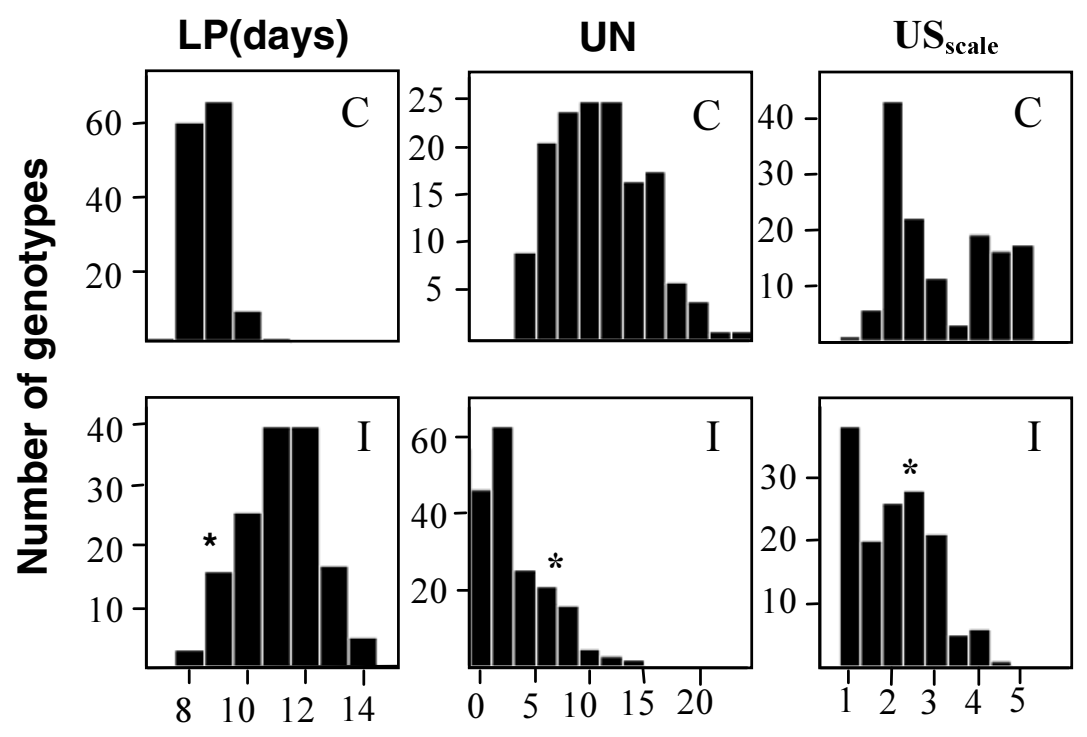

Fig. 4. Distribution of genotypic means for latent period (LP), uredinia number (UN) 13 days after inoculation, and uredinia size score (US scale $) 14$ days after inoculation in 336 Populus deltoides $\times$ P. trichocarpa full-sib clones inoculated with Melampsora larici-populina single uredinial strain 93CV1 at 30-mg/liter inoculum pressure level in a 5-complete-block design. Data are presented separately for the 145 clones compatible (C) with M. larici-populina strain 93ID6 and for the 191 clones incompatible (I) with this strain. The observed clonal means for the $P$. trichocarpa male parent are represented by an asterisk. 
genotype-strain interactions assessments by facilitating comparison between experiments involving different M. larici-populina strains.

The present study yields one important result regarding sensitivity of partial resistance components expression to inoculum pressure level. A doubling of the inoculum pressure level from 30 to $60 \mathrm{mg} /$ liter (from 56 to 112 urediniospores per leaf disk) had no

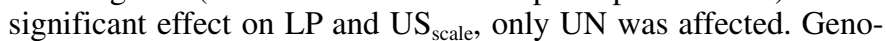
type-inoculum pressure level effect on UN did not affect the relative ranking of genotypes significantly, it only stressed the difference between highly susceptible versus highly resistant subpopulations. The optimum inoculum pressure level for quantification of genetic variation for partial resistance components should be a compromise between the need to avoid false complete resistance response and easiness of measure on numerous genotypes.

Measuring LP, UN 13 days after inoculation, and US $_{\text {scale }} 14$ days after inoculation on excised leaf disks inoculated with a given $M$. larici-populina strain at inoculum pressure between 60 and 120 urediniospores per leaf disk proves to be effective for precise description of genetic variation for partial resistance. However, even with this technique, all environmental conditions cannot be easily controlled when dealing with high numbers of genotypes. Expected confounding of both environmental (such as inoculum pressure, preinoculation temperature and shoot age) and strain effects leads us to expect future difficulties when attempting to assess genotype-strain interactions from comparison of independent mono-strain experiments.

Observed genetic variability. Working on a relatively large population made it possible to gain insight into the genetic determinism of complete resistance to $M$. larici-populina strain $93 \mathrm{CV} 1$. In the same interspecific family, Lefèvre et al. (8) suggested a digenic model for complete resistance to 93CV1 with the following three restrictive conditions: (i) the two loci should be tightly linked in a single cluster, (ii) genes interact in such a way that dominant alleles are needed at both loci to obtain a resistant phenotype, and (iii) the $P$. deltoides parent (completely resistant) should be double heterozygous in repulsing phase and the $P$. trichocarpa parent (susceptible) should be double recessive homozygous. Five of the fourty-nine $F_{1}$ genotypes that behaved as completely resistant to $93 \mathrm{CV} 1$ in this experiment confirmed their incompatibility under repeated high inoculum pressure (data not
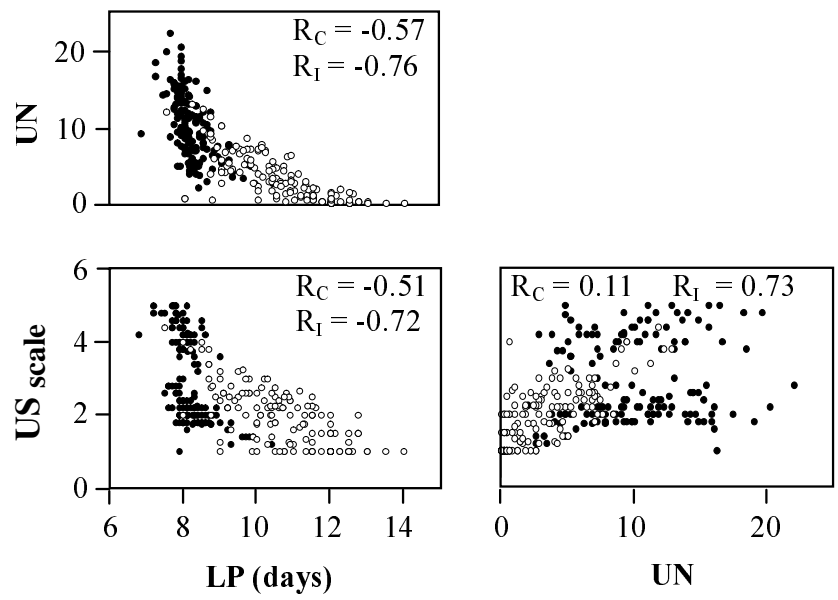

Fig. 5. Relationships between genotypic means for latent period (LP), uredinia number (UN) 13 days after inoculation, and uredinia size score $\left(\mathrm{US}_{\text {scale }}\right) 14$ days after inoculation for 336 Populus deltoides $\times P$. trichocarpa full-sib clones inoculated with Melampsora larici-populina single uredinial strain 93CV1 at 30-mg/liter inoculum pressure level in a 5-complete-block design. Clones compatible with $M$. larici-populina strain 93ID6 appear as black dots and those incompatible with this strain appear as open circles. Pearson's correlation coefficients are represented in the upper right corner of each plot for the compatible $\left(\mathrm{R}_{\mathrm{C}}\right)$ and the incompatible $\left(\mathrm{R}_{\mathrm{I}}\right)$ clones. shown), whereas no incompatible genotype had been identified in the smaller population studied by Lefèvre et al. (8). With $1.5 \%$ favorable recombinants, this result is consistent with the model hypothesized for complete resistance to strain 93CV1. Triploidy, which has been demonstrated in $P$. trichocarpa $\times P$. deltoides hybrids (2), could also explain the presence of incompatible genotypes.

In the present study, subpopulations of genotypes compatible versus incompatible with strain 93ID6 were large enough to be compared and thus, allowed for better comprehension of the genetic relationship between partial and complete resistance. Presence of complete resistance against strain 93ID6 went together

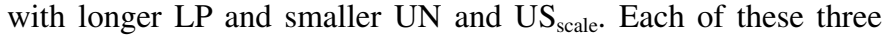
key parameters of $M$. larici-populina epidemics may be influenced independently. However, we also suspect an indirect effect on UN and $\mathrm{US}_{\text {scale }}$ through longer LP: triangular relationships between LP and both UN and $\mathrm{US}_{\text {scale }}$ in the incompatible subpopulation may result from the limiting effect of very long LP on the expression of the two other traits. When LP was less than 10 days, either in absence or in presence of complete resistance to strain 93ID6, correlations between LP and both UN and $\mathrm{US}_{\text {scale }}$ were obviously lower. Moderate to high correlations between partial resistance components are often reported in different host-pathogen interactions $(3,9,17,18,22)$. There is no evidence whether the observed relationship between presence of complete resistance to strain 93ID6 and the level of partial resistance to strain 93CV1 comes from pleiotropy or linkage. Three nonexclusive hypothesis can be formulated. (i) Studies on another $P$. deltoides $\times P$. trichocarpa $\mathrm{F}_{1}$ progeny where there is cosegregation for complete resistance to strains 93ID6 and 93CV1 led to the conclusion that one of the two interacting genes responsible for complete resistance to strain 93CV1 is tightly clustered with one gene involved in complete resistance to 93ID6 (8). This gene, when interaction is broken, may have an effect on partial resistance expression. (ii) Other genes tightly clustered with one gene controlling complete resistance to 93ID6 may also be in cause. (iii) The dominant allele at locus controlling complete resistance to 93ID6 may have residual effects on partial resistance. Under the first and second hypotheses, two categories of recombinants are expected: genotypes compatible with 93ID6 with strong partial resistance to 93CV1 and genotypes incompatible with 93ID6 with weak partial resistance to 93CV1. A few genotypes within the 336 tested $F_{1}$ could indeed correspond to such recombinants but their behavior must be confirmed. Under the third hypothesis, "defeated" complete resistance genes could act as partial resistance genes against a virulent $M$. larici-populina strain. This model was effective in the rice-Xanthomonas oryzae pv. oryzae model (13).

The significantly large subpopulation of genotypes compatible with strain 93ID6 is informative about the expression of partial resistance components in a genetic background where no complete resistance to $M$. larici-populina has been detected so far. A polygenic determinism can be inferred from the unimodal distributions of clonal means of both LP and UN. However, bimodal distribution of clonal means for $\mathrm{US}_{\text {scale }}$ and significant residual genetic variability within the two $\mathrm{US}_{\text {scale }}$ classes are indicative of a major gene effect associated with ancillary, or minor, gene effects. This result is similar to conclusions from Newcombe (15), who found combined effects of major and minor genes in the genetic determinism of partial resistance of $P$. trichocarpa $\times P$. deltoides hybrids to $M$. medusae f. sp. deltoidae. This type of model is often detected when studying plant partial resistance $(4,11,12,19,30)$. Bimodality for uredinia size was also observed in four intraspecific $P$. trichocarpa $\times P$. trichocarpa $\mathrm{F}_{1}$ families involving the same male parent and inoculated with strain 93CV1 (23). We can hence infer that this gene is inherited from the $P$. trichocarpa male parent, demonstrating existence of exapted resistance to a disease in Populus spp. (16). It also demonstrates that this major locus that controls partial resistance in the pure species $P$. trichocarpa is 
still effective in interspecific $P$. deltoides $\times P$. trichocarpa hybrids where no complete resistance to $M$. larici-populina has been detected so far. It may also be effective in the interspecific hybrids expressing complete resistance, in association with the presenceof-complete-resistance-genes effect. Ongoing genotyping using associated markers will help answer this question.

Two major genes involved in partial resistance to strain 93CV1 have been identified in this study. One is inherited from the $P$. deltoides parent and associated with complete resistance to strain 93ID6, the other one is inherited from the P. trichocarpa parent. However, they did not account for all the observed genetic variability for the three epidemiological components. More refined genetic dissection of these quantitative variations would benefit from quantitative trait loci analyses. A multiple progeny approach is now needed to identify other loci that show polymorphism in each parental species. Association of different $P$. trichocarpa partial resistance genes in different $P$. deltoides genetic backgrounds (i.e., absence or different combinations of complete resistance genes) would also provide information on potential favorable gene interactions, a prerequisite for constructing complex resistance in poplars.

\section{ACKNOWLEDGMENTS}

Financial support was provided by grants from INRA, Région Centre, and the French Ministry of Agriculture and Fisheries. We thank D. Lacan, P. Poursat, and their collaborators at the Unité Expérimentale of INRA Orléans for excellent technical assistance; and V. Jorge, F. Lefèvre, and two anonymous reviewers for their valuable comments on the manuscript.

\section{LITERATURE CITED}

1. Box, G. E. P., and Cox, D. R. 1964. The analysis of transformations (with discussion). J. R. Stat. Soc. 26:211-252.

2. Bradshaw, H. D., and Stettler, R. F. 1993. Molecular genetics of growth and development in Populus. I. Triploidy in hybrid poplars. Theor. Appl. Genet. 86:301-307.

3. Broers, L. H. M. 1989. Influence of development stage and host genotype on three components to resistance to leaf rust in spring wheat. Euphytica 44:187-190.

4. Caranta, C., Lefebvre, V., and Pallois, A. 1997. Polygenic resistance of pepper to potyviruses consists of a combination of isolate-specific and broad-spectrum quantitative loci. Mol. Plant-Microbe Interact. 10:872-878.

5. Chandrashekar, M., and Heather, W. A. 1981. The effect of pre- and postinoculation temperature on resistance in certain cultivars of poplar to races of Melampsora larici-populina Kleb. Euphytica 30:113-120.

6. Goué-Mourier, M. C., Faivre-Rampant, P., Le Guerroué, B., Lefèvre, F., and Villar, M. 1996. Molecular and genetic approaches to rust resistance (Melampsora sp.) in Poplar (Populus sp.). Pages 249-254 in: Somatic Cell Genetics and Molecular Genetics of Trees. M. R. Ahuja, W. Boerjan, and D. B. Neale, eds. Kluwer Academic Publishers, Dordrecht, The Netherlands.

7. Johnson, R. 1984. A critical analysis of durable resistance. Annu. Rev. Phytopathol. 22:309-330.

8. Lefèvre, F., Goué-Mourier, M. C., Faivre-Rampant, P., and Villar, M. 1998. A single gene cluster controls incompatibility and partial resistance to various Melampsora larici-populina races in hybrid poplars. Phytopathology 88:156-163.

9. Lefèvre, F., Pichot, C., and Pinon, J. 1994. Intra- and interspecific in- heritance of some components of the resistance to leaf rust (Melampsora larici-populina Kleb.) in poplars. Theor. Appl. Genet. 88:501-507.

10. Lemaire, H., Maugard, F., and Merzeau, D. 1998. Faut-il traiter les peupliers contre la rouille? Essais de traitements sur Luisa Avanzo en Aquitaine. Forêt Entreprise 121:54-59.

11. Leonards-Schippers, C., Gieffers, W., Schäfer-Pregl, R., Ritter, E., Knapp, S. J., Salamini, F., and Gebhardt, C. 1994. Quantitative resistance to Phytophthora infestans in potato: A case study for QTL mapping in allogamous plant species. Genetics 137:67-77.

12. Lespinasse, D., Grivet, L., Troispoux, V., Rodier-Goud, M., Pinard, F., and Seguin, M. 2000. Identification of QTLs involved in the resistance to South American leaf blight (Microcyclus ulei) in the rubber tree. Theor. Appl. Genet. 100:975-984.

13. Li, Z. K., Luo, L. J., Mei, H. W., Paterson, A. H., Zhao, X. H., Zhang, D. B., Wang, Y. P., Yu, X. Q., Zhu, L., Tabien, R., Stansel, J. W., and Ying, C. S. 1999. A "defeated" rice resistance gene acts as a QTL against a virulent strain of Xanthomonas oryzae pv. oryzae. Mol. Gen. Genet. 261:58-63.

14. Messmer, M. M., Seyfarth, R., Keller, M., Schachermayr, G., Winzeler, M., Zanetti, S., Feuillet, C., and Keller, B. 2000. Genetic analysis of durable leaf rust resistance in winter wheat. Theor. Appl. Genet. 100:419431.

15. Newcombe, G. 1998. Association of Mmdl, a major gene for resistance to Melampsora medusae f. sp. deltoidae, with quantitative traits in poplar rust. Phytopathology 88:114-121.

16. Newcombe, G. 1998. A review of exapted resistance to disease of Populus. Eur. J. For. Pathol. 28:209-216.

17. Ohm, H. W., and Shaner, G. E. 1976. Three components of slow leaf rusting at different growth stages in wheat. Phytopathology 66:13561360 .

18. Parlevliet, J. E. 1979. Components of resistance that reduce the rate of epidemic development. Annu. Rev. Phytopathol. 17:203-222.

19. Pecchioni, N., Faccioli, P., Toubia-Rahme, H., Valè, G., and Terzi, V. 1996. Quantitative resistance to barley leaf stripe (Pyrenophora graminea) is dominated by one major locus. Theor. Appl. Genet. 93:97-101.

20. Pinon, J., and Frey, P. 1997. Structure of Melampsora larici-populina populations on wild and cultivated poplar. Eur. J. Plant Pathol. 103:159-173.

21. Pinon, J., Newcombe, G., and Chastagner, G. A. 1994. Identification of races of Melampsora larici-populina, the Eurasian rust fungus, on Populus species in California and Washington. Plant Dis. 78:101.

22. Rashid, K. Y. 1991. Evaluation of components of partial resistance to rust in flax. Can. J. Plant Pathol. 13:212-217.

23. Saintagne, C., Faivre Rampant, P., Bastien, C., Goué, M. C., Lacan, D., Lesage, M. C., Villar, M., and Prat, D. 1999. Detection of QTLs in Populus trichocarpa linked with partial resistance to foliar rust Melampsora larici-populina (race E2). Page 100 in: Proc. IUFRO Int. Poplar Symp. II.

24. Shaner, G., and Hess, F. D. 1978. Equations for integrating components of slow-rusting resistance in wheat. Phytopathology 68:1464-1469.

25. Sharma, J. K., and Heather, W. A. 1978. Parasitism of uredospores of Melampsora larici-populina Kleb. by Cladosporium sp. Eur. J. For. Pathol. 8:48-54.

26. Sharma, J. K., and Heather, W. A. 1979. Comparison of disease parameters for quantitative assessment of Melampsora leaf rust in clones of Populus spp. Trans. Brit. Mycol. Soc. 72:483-488.

27. Sharma, J. K., Heather, W. A., and Winer, P. 1980. Effect of leaf maturity and shoot age of clones of Populus species on susceptibility to Melampsora larici-populina. Phytopathology 70:548-554.

28. Van Kraayenoord, C. W. S., Laudon, G. F., and Spiers, A. G. 1974. Poplar rusts invade New Zealand. Plant Dis. Rep. 58:423-427.

29. Walker, J., Hartigan, D., and Bertus, A. L. 1974. Poplar rusts in Australia with comments on potential conifer rusts. Eur. J. For. Pathol. 4:100-118.

30. Young, N. D. 1996. QTL mapping and quantitative disease resistance in plants. Annu. Rev. Phytopathol. 34:479-501. 